\title{
BMJ Open A continuous PREMs and PROMs Observatory for elective hip and knee arthroplasty: study protocol
}

\author{
Sabina De Rosis (D) , ${ }^{1}$ Francesca Pennucci (D) , ${ }^{1}$ Daniel Adrian Lungu (D) , \\ Mario Manca, ${ }^{2}$ Sabina Nuti (i) ${ }^{1}$
}

To cite: De Rosis S,

Pennucci F, Lungu DA, et al.

$A$ continuous PREMs and

PROMs Observatory for elective

hip and knee arthroplasty:

study protocol. BMJ Open

2021;11:e049826. doi:10.1136/

bmjopen-2021-049826

- Prepublication history for this paper is available online. To view these files, please visit the journal online (http://dx.doi. org/10.1136/bmjopen-2021049826).

Received 03 February 2021 Accepted 15 July 2021

Check for updates

(C) Author(s) (or their employer(s)) 2021. Re-use permitted under CC BY-NC. No commercial re-use. See rights and permissions. Published by BMJ.

${ }^{1}$ Management and Healthcare Laboratory, Institute of Management and Department EMbeDS, Scuola Superiore Sant'Anna, Pisa, Italy

${ }^{2}$ Department of Orthopaedic

Surgery, Versilia Hospital, Lido di Camaiore, Italy

Correspondence to Dr Francesca Pennucci; f.pennucci@santannapisa.it

\section{ABSTRACT}

Introduction Scholars, healthcare practitioners and policymakers have increasingly focused their attention on patient-centredness. Patient-reported metrics support patient-driven improvement actions in healthcare systems. Despite the great interest, patient-reported outcome measures (PROMs) are still not extensively collected in many countries and not integrated with the collection of patient-reported experience measures (PREMs). This protocol describes the methodology behind an innovative observatory implemented in Tuscany, Italy, aiming at continuously and longitudinally collecting PROMs and PREMs for elective hip and knee total replacement. Methods and analysis The Observatory is digital. Enrolled patients are invited via SMS or email to online questionnaires, which include the Oxford Hip Score or the Oxford Knee Score. Data are real-time reported to healthcare professionals and managers in a raw format, anonymised and aggregated on a web platform. The data will be used to investigate the relationship between the PROMs trend and patients' characteristics, surgical procedure, hospital characteristics, and PREMs. Indicators using patient data will be computed, and they will integrate the healthcare performance evaluation system adopted in Tuscany.

Ethics and dissemination The data protection officers of local healthcare organisations and the regional privacy office framed the initiative referring to the national and regional guidelines that regulate patient surveys. The findings will be reported both in real time and for publication in peer-reviewed journals.

\section{INTRODUCTION}

This paper describes the protocol of the Orthopaedic PROMs and PREMs Observatory (OPPO) on elective total hip replacement (THR) and total knee replacement (TKR) surgery. Despite this not being a clinical trial protocol, the authors found it useful to follow the Standard Protocol Items: Recommendations for Interventional Trials (SPIRIT)-PRO Extension checklist. ${ }^{1}$

\section{Roles and responsibilities}

The funder has been periodically informed on the design and implementation of the study. It does not have ultimate authority

\section{Strengths and limitations of this study}

- This protocol describes the first regional-level, digital, and continuous patient-reported outcome measures (PROMs) and patient-reported experience measures (PREMs) observatory on total hip replacement and total knee replacement in Italy.

- This PROMs and PREMs digital data collection system is low cost and easy to use and allows for a real-time reporting of data to providers.

- Since the role of providers is key in this system, selection bias and potential low participation rates need to be constantly monitored.

- Elderly patients can have difficulties in participating; therefore, caregivers are also involved to help in accessing and filling in the questionnaires.

over the collection, management, analysis, interpretation of data, and writing of reports. The coordinating centre is the Management and Healthcare (MeS) Laboratory of the Sant'Anna School of Pisa, which oversees the methodology definition, the study implementation, as well as the data collection, management, and reporting.

The study has been implemented by involving all Tuscan public healthcare providers, which are the data owners and are involved in data collection, interpretation and use.

\section{BACKGROUND}

Osteoarthritis (OA) is a common and debilitating disease with a prevalence ranging from $12.3 \%$ to $21.6 \%$, according to different studies. $^{2}$ OA is among the main causes of disability and absence from work in most Western countries, implying high costs of care and a relevant social impact. ${ }^{3}$ In Italy, hip pain affects around $12 \%$ of people aged 65 and older. ${ }^{4}$ In 2017, OA was the fifth most frequent diagnosis. ${ }^{5}$

There is evidence on the cost-effectiveness of THR and TKR; these procedures have been 
recommended for patients with end-stage or severe OA. ${ }^{6}$ In 2017, in Italy, there were more than 172000 hospital discharges for knee replacements and more than 108000 for hip replacements, excluding day surgery; average hospital stay was around 9 days. ${ }^{5}$

Ageing of the population affects the prevalence of $\mathrm{OA}^{7}$ and, consequently, increases the costs associated with THR and TKR. ${ }^{8}$ The relevance of ensuring a good and quick recovery of functionalities and quality of life to patients undergoing elective arthroplasties is self-evident. Positive results for the patients have beneficial implications for the healthcare system as well. The economic implications are related not only on the duration of the hospital stay alone but also on the care pathway after surgery (ie, rehabilitation). These aspects are relevant in public healthcare systems, since resources are limited and have an impact also on society, for example, in terms of indirect costs. ${ }^{9}$ This last key perspective is still lacking at a system policymaking level, ${ }^{6}$ while the most commonly adopted perspective is solely the healthcare cost.

Several elements can impact the short-term outcomes after THR and TKR surgery. Physiotherapy is an accepted standard of care after total joint arthroplasty in order to maximise joint functionality and minimise complication risks. ${ }^{10}$ However, there is mixed evidence on the rehabilitation outcomes. It has been shown that clinical pathways can significantly improve the quality of care during hospitalisation for arthroplasty. ${ }^{11}$ Despite the efforts carried on in different countries, ${ }^{12}{ }^{13}$ clinical evidence-based guidelines about appropriate postdischarge pathway are not well defined or available. ${ }^{10}$

Another important aspect to consider is related to expectations and awareness of patients undergoing arthroplasty. Indeed, expectations and awareness are part of the appropriateness criteria for arthroplasty that require the elicitation of the patient's knowledge and preferences. ${ }^{14}$ Generally, patients tend to overestimate potential benefits of surgery and underestimate potential complication risks. ${ }^{15}$ In addition, decision-making processes, even if supported by decision aids, are generally based on clinically focused outcomes (eg, surgical mortality or complications) and not on other outcomes such as pain or mobility, which are often priorities for patients. ${ }^{16}$

According to the patient-centredness care, to support the shift from volumes to value, it is crucial to investigate the impact of THR and TKR in the patients' perspective. To this end, patient-reported outcome measures (PROMs) aim to investigate if a healthcare intervention made, or not, a difference in the patient's health condition, quality of life and/or other aspects that are relevant to patients. ${ }^{17}$ PROMs can respond to the needs of different stakeholders: patients themselves, clinicians, decision makers, and policymakers. ${ }^{18-20}$ PROMs can be used for assessing care quality and benchmarking healthcare service providers by providing a measure of outcomes produced along the care pathway. ${ }^{21}$
Patient-reported experience measures (PREMs) are a complementary source of information of the patients' view on service delivery. PREMs intend to elicit what actually occurred to patients while receiving care, providing managers and professionals with specific information on 'what does not work' and 'what works' in the patients' perspective. ${ }^{22}$

At the international level, there are several experiences of PROMs and PREMs collection and reporting. ${ }^{22-27}$ Nevertheless, the different initiatives are in general patchy or not continuous, while several countries have not yet adopted any of these tools. ${ }^{22}$ Most of the initiatives focus alternatively only on PROMs or PREMs, thus not allowing for a longitudinal monitoring of care coordination and integration along the patient care pathway, ${ }^{28}$ or do not integrate them with data from other sources. ${ }^{22}{ }^{29-31}$ The Organisation for Economic Co-operation and Development has been working with several countries for systematically collecting comparable PROM data for different care pathways,${ }^{32}$ including joint replacement: 10 among national (England, Netherlands and Sweden) as well as regional (Canada-Alberta and Manitoba, SwitzerlandGeneva) initiatives, subnational registries (the Australian Clinical Outcomes Registry, which collects data from providers in two states) and single hospitals (Coxa Hospital, Finland, and the Galeazzi Institute, Italy). ${ }^{33}$ Despite the self-evident value of international comparison and the proactivity of the orthopaedic community in collecting patient-reported data, reporting PROM information alone seems to be insufficient to identify opportunities for quality improvement, ${ }^{28}$ also in terms of inclusion of patient-reported indicators in performance evaluation systems (PESs). ${ }^{34}$ National-level reporting is still an exception, and often patient-reported data collections are patchy, locally developed or voluntary-based. ${ }^{33}$

In the Italian scenario, there are multiple experiences of PROM collection, mainly within clinical trials or experimental studies. Instead, there are no examples of ongoing systematic collections of PROMs integrated with PREMs, and no integration of PROMs and PREMs with data from other sources within PESs. The PREM and PROM observatory implemented in Tuscany (central Italy) is the first example of a regional-level, digital, standardised and continuous collection of PROMs and PREMs in Italy for multiple care pathways ${ }^{18}$ : breast cancer surgery $^{3536}$; prostate, colorectal and lung cancer robotic surgery $^{37}$; chronic heart failure ${ }^{38-40}$; maternal pathway ${ }^{41}$; and orthopaedic elective surgery (THR and TKR).

\section{METHODS}

\section{Study setting}

The OPPO is implemented in Tuscany. In 2017, Tuscan public hospitals provided a total of 5728 THRs and TKRs, while in 2018 these were 6099. In 2018, hospitalisation rates for hip replacements, excluding traumas, varied between 265 and 467 interventions per 100000 inhabitants. Figure 1 shows an increasing trend over time. 


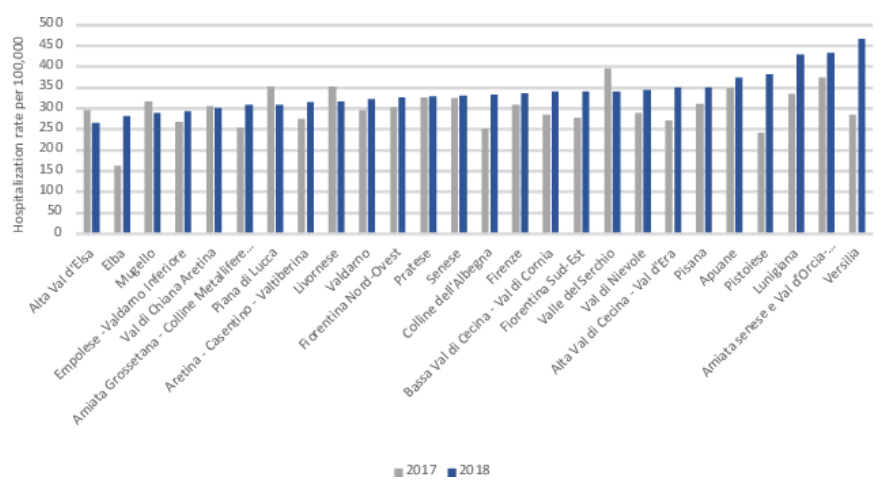

Figure 1 Standardised hospitalisation rate and trend for hip replacement interventions for 100000 residents. Period: 2017-2018. Source: Performance Evaluation System (www. performance.sssup.it/netval)

Considering the knee replacement interventions in the adult population, excluding traumas, the global rate was around 171 interventions per 100000 inhabitants in 2018, with extensive variation among districts (min 108$\max 245$ ) (figure 2).

In Tuscany, as well as in other Italian regions, waiting times for hip and knee replacement surgeries are highly variable among hospitals. Considering the classification of interventions between priority classes (A, B and $\mathrm{C}$, in order of urgency), the waiting times vary from a few days to over 18 months.

The OPPO has been implemented by involving all Tuscan public healthcare providers performing THR and TKR surgeries, namely, three local health authorities with multiple hospitals and three teaching hospitals. In 2019, the active study sites were 16 public hospitals (table 1 ).

\section{Design of the study}

The OPPO is a digital, systematic and continuous initiative based on a prospective, multisite cohort study. The surveys encompassed by the OPPO are longitudinal, and standardised PROM and PREM data are digitally collected and reported on a web platform, which is currently linked neither to the electronic medical records of patients nor to the Registry of Orthopaedic Prosthetic Implants yet.

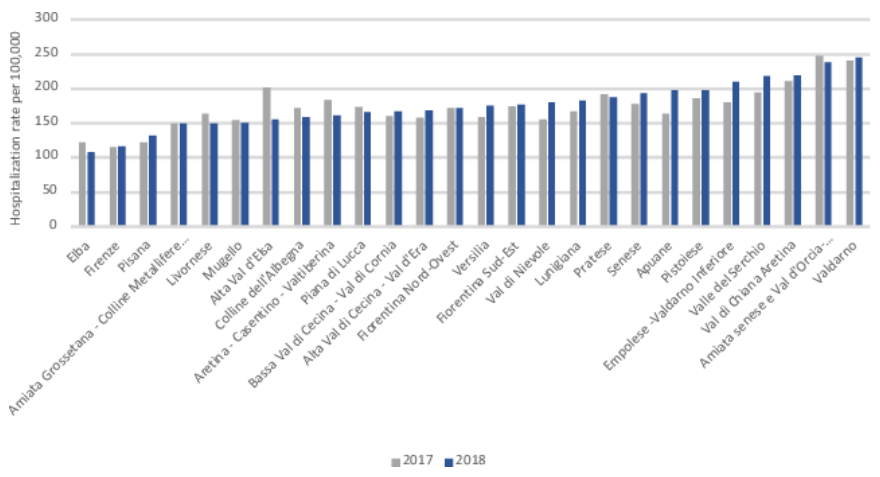

Figure 2 Standardised hospitalisation rate and trend for knee replacement interventions for 100000 residents. Period: 2017-2018. Source: Performance Evaluation System (www. performance.sssup.it/netval).
PROMs and PREMs questionnaires are electronically administered to patients. Within 24 hours from the presurgery enrolment, patients receive a Short Message Service (SMS) and/or an email containing a personal link to the web questionnaire. The choice of the digital methodology has been made for several reasons: a growing confidence with smartphones and tablets among the elderly people and increasing evidence on their possibility to participate in online surveys, ${ }^{42-44}$ cost-effectiveness, timeliness as data are real-time collected and reported, and the decreasing use of landline phones and regular mail. Currently, the digital methodology could represent a barrier for some older adults due to digital divide or low digital skills. For mitigating the potential bias of selection that this can lead, the enrolment of patients can encompass the involvement of their caregiver, if any, who can help the patient to receive, access and reply to the questionnaire. The involvement of caregivers will help also in overcoming physical limitations (eg, poor eyesight, reduced mobility, and other conditions which can affect understanding) or other barriers to accessing the questionnaire (eg, not having a smartphone, tablet or computer, or not having access to the internet). Since caregivers might have an influence on the responses given by patients, the questionnaire collects the information on the presence of a caregiver helping the patient in participating in the survey. The follow-up questionnaires are electronically sent 1, 6 and 12 months after surgery. Patients can fill the questionnaire autonomously anywhere, without the presence of a healthcare worker who might influence them.

Participation and retention are promoted directly by professionals, during encounters, by communication materials (ie, posters and leaflets) in hospitals and ambulatories, and by SMS and email reminders automatically sent to enrolled patients.

\section{Eligibility criteria and recruitment}

The population of enrolees include consecutive patients undergoing elective THR or TKR admitted to the 19 operative units of 16 public hospitals (table 1 ).

The eligibility criteria comprise patients who received a surgical indication and for whom surgery has been therefore scheduled. The inclusion criteria are

- Male and female genders.

- Any age. ${ }^{45}$

- Elective intervention with the International Classification of Diseases, 9th revision - Clinical Modification (ICD-9-CM) codes 81.51 for THR and 81.54 for TKR.

- Use of unicompartmental, bicompartmental or tricompartmental prostheses.

The willingness to be enrolled is another participation criterion to the OPPO. Eligible patients are informed by orthopaedic professionals and their staff: (1) in an ambulatory setting, during the presurgical visit when surgery is scheduled; (2) in the hospital, during the preadmission phase, which is generally 30 days before surgery; or (3) directly in the ward before surgery. If the patient explicitly accepts to participate in the initiative, orthopaedic 
Table 1 Hospitals and operative units joining the Orthopaedic PROMs and PREMs Observatory for elective orthopaedic surgery in Tuscany (Italy)

\begin{tabular}{|c|c|c|c|c|}
\hline & $\begin{array}{l}\text { Healthcare } \\
\text { organisation }\end{array}$ & Hospital & $\begin{array}{l}\text { Operative } \\
\text { units }\end{array}$ & THR and TKR 2019 (n) \\
\hline \multirow[t]{3}{*}{ THs } & $\begin{array}{l}\text { Azienda Ospedaliera } \\
\text { Universitaria Pisana }\end{array}$ & AOU Pisana & 3 & 516 \\
\hline & $\begin{array}{l}\text { Azienda Ospedaliera } \\
\text { Universitaria Senese }\end{array}$ & AOU Senese & 1 & 273 \\
\hline & $\begin{array}{l}\text { Azienda Ospedaliera } \\
\text { Universitaria Careggi }\end{array}$ & AOU Careggi & 2 & 719 \\
\hline \multirow[t]{10}{*}{ LHAs } & Ausl Nord Ovest & $\begin{array}{l}\text { Ospedale Massa, Nuovo Ospedale delle } \\
\text { Apuane }\end{array}$ & 1 & 104 \\
\hline & & $\begin{array}{l}\text { Ospedale Castelnuovo di Garfagnana, } \\
\text { Santa Croce, Lucca }\end{array}$ & 1 & 170 \\
\hline & & Ospedale Pontedera, Felice Lotti & 1 & 308 \\
\hline & & Ospedale Unico Versilia, Viareggio & 1 & 318 \\
\hline & Ausl Centro & Ospedale Torregalli, San Giovanni di Dio & 1 & 88 \\
\hline & & Ospedale Empoli, San Giuseppe & 1 & 120 \\
\hline & & Ospedale Fucecchio, San Pietro Igneo & 1 & 908 \\
\hline & Ausl Sud Est & Ospedale Arezzo, San Donato & 1 & 211 \\
\hline & & Ospedale Misericordia Grosseto & 1 & 122 \\
\hline & & $\begin{array}{l}\text { Ospedale Cortona, Fratta, Valdichiana } \\
\text { Santa Margherita }\end{array}$ & 1 & 290 \\
\hline
\end{tabular}

LHA, local health authority; TH, teaching hospital; THR, total hip replacement; TKR, total knee replacement.

professionals and their team collect the patient's preliminary information on the aforementioned web platform.

The exclusion criteria include

- Patients operated due to fracture or trauma with urgent surgery.

- Patients who undergo surgery for prosthesis revision and partial hip or knee replacement, indicated by the procedure code ICD-9-CM 85.12.

- Patients with severe cognitive impairment, evaluated by the clinician using proper tools (eg, Mini-Mental State Examination tool). These patients are excluded because of their inability to reply to the questionnaire, although including them would be important in the future by using different approaches. ${ }^{46}$

- Arthroplasty for musculoskeletal cancer.

- Non-eligibility for surgical procedures.

\section{Sample size and duration}

OPPO's aim is to systematically include all patients meeting the eligibility criteria and undergoing elective hip and knee arthroplasty in Tuscany. Each patient undergoing a THR or a TKR can participate. According to previous findings on participation in longitudinal studies, ${ }^{478}$ the expected enrolment rate is approximately $30 \%$ of patients in the first year of the OPPO. Given that in 2018 there were 2362 THRs and 2143 TKRs, the expectation was to enrol approximately 1351 patients: 708 for THR and 643 for TKR. An increase in participation rate is possible over time, in case of improved familiarity of people with digital surveys, ${ }^{44}$ increased familiarity with the initiative itself, and increased willingness and motivation of clinicians. The participation rate will be constantly monitored by the research team to promptly intervene in case a specific group of patients (eg, cared by the same clinician, same age group, etc) will significantly decrease their participation rate under the threshold of $30 \%$. Since previous studies showed a drop-out rate at follow-up ranging between $30 \%$ and $70 \%,{ }^{49}$ a follow-up drop-out rate of around $50 \%$ is expected.

The OPPO was designed as a routine system without a predefined ending date.

\section{Outcomes}

Primary outcome measures are related to functionality, pain and quality of life, and experience with care along the pathway. They are collected using PROMs and PREMs questionnaires, chosen with professionals after conducting an initial literature review.

The disease-specific PROMs questionnaires used for THR and TKR are, respectively, the Oxford Hip Score (OHS) and the Oxford Knee Score (OKS), provided by the Oxford University Innovation Limited. ${ }^{50-54}$ The 
Italian version of the OKS was validated directly by the Oxford University. An Italian version of the OHS has been validated through an Italian study. ${ }^{55}$ The Italian version used for OPPO included some minor improvements, which were shared and agreed directly with the Oxford University during the process of licence granting of OHS and OKS questionnaires.

These scales are standardised and widely used, therefore allowing an international comparison of PROM results from the Tuscan OPPO. Moreover, they were chosen because of their relative brevity (12 questions), the inclusion of several relevant domains, and their validity in relation to other generic PROMs questionnaires and clinician-reported questionnaires. ${ }^{57} 58$ The domains investigated by the OHS and the OKS are pain (ie, walking and standing up from a chair); motor skills (ie, ability to go up/downstairs and wear socks); and daily life activities (ie, ability to wash and dry themselves).

The preoperative and postoperative questionnaires contain PREMs questions designed to detect the patient experience throughout the care pathway (table 2).

Table 2 Sociodemographic, experience and additional outcome questions included into the Orthopaedic PROMs and PREMs Observatory questionnaires by timepoint

\begin{tabular}{|c|c|c|c|c|}
\hline Questionnaire & $\begin{array}{l}\text { Timing of } \\
\text { administration }\end{array}$ & Rationale & $\begin{array}{l}\text { Dimensions of } \\
\text { PREMs }\end{array}$ & Items \\
\hline \multirow[t]{3}{*}{$\begin{array}{l}\text { First questionnaire } \\
\text { (preoperative or T0) }\end{array}$} & \multirow[t]{3}{*}{$\begin{array}{l}\text { Before surgery (at } \\
\text { surgical indication } \\
\text { or preadmission) }\end{array}$} & \multirow[t]{2}{*}{$\begin{array}{l}\text { To characterise } \\
\text { patients and ensure the } \\
\text { comparability of data } \\
\text { through risk adjustment } \\
\text { procedures }\end{array}$} & $\begin{array}{l}\text { Sociodemographic } \\
\text { information }\end{array}$ & $\begin{array}{l}\text { Age. } \\
\text { Weight and height to calculate the body mass index. } \\
\text { Level of education. } \\
\text { Marital status. } \\
\text { Cohabitation. } \\
\text { Social support. } \\
\text { Job (current or past). }\end{array}$ \\
\hline & & & $\begin{array}{l}\text { Clinically related } \\
\text { characteristics of the } \\
\text { patient }\end{array}$ & $\begin{array}{l}\text { Comorbidities. } \\
\text { Use of psychotropic drugs. } \\
\text { Use of aids. }\end{array}$ \\
\hline & & $\begin{array}{l}\text { To map the process that } \\
\text { led the patient to decide to } \\
\text { receive surgery }\end{array}$ & $\begin{array}{l}\text { Experience with } \\
\text { services }\end{array}$ & $\begin{array}{l}\text { Visits and examinations before surgery. } \\
\text { Sources of information (ie, general practitioner, } \\
\text { professional and internet). } \\
\text { Role of the general practitioner. } \\
\text { Second medical opinion about the surgical } \\
\text { indication. }\end{array}$ \\
\hline \multirow[t]{2}{*}{$\begin{array}{l}\text { First postoperative } \\
\text { questionnaire (T1) }\end{array}$} & \multirow[t]{2}{*}{$\begin{array}{l}30 \text { days after } \\
\text { surgery }\end{array}$} & $\begin{array}{l}\text { To identify experiential } \\
\text { factors affecting the } \\
\text { outcomes; the questions } \\
\text { are developed on the } \\
\text { basis of a still used PREM } \\
\text { questionnaire }\end{array}$ & $\begin{array}{l}\text { Hospitalisation } \\
\text { experience }\end{array}$ & $\begin{array}{l}\text { Admission to the hospital, facility comfort. } \\
\text { Communication and involvement in decision-making. } \\
\text { Emotional support received. } \\
\text { Complications and adverse events during the } \\
\text { hospital stay. } \\
\text { Clarity and completeness of information at discharge. } \\
\text { Satisfaction with the care. } \\
\text { Willingness to recommend. } \\
\text { Open-ended question. }\end{array}$ \\
\hline & & $\begin{array}{l}\text { To map the pathway before } \\
\text { and after surgery and } \\
\text { identify experiential factors } \\
\text { affecting the outcomes }\end{array}$ & $\begin{array}{l}\text { Experience with } \\
\text { services }\end{array}$ & $\begin{array}{l}\text { Waiting time before intervention. } \\
\text { Hospital-community/primary care continuity. } \\
\text { Role of the general practitioner. } \\
\text { Open-ended question on the care pathway before } \\
\text { surgery. } \\
\text { Rehabilitation services. } \\
\text { Early rehabilitative practices during hospital stay and } \\
\text { after discharge. } \\
\text { Open-ended question on the care pathway after } \\
\text { discharge. }\end{array}$ \\
\hline $\begin{array}{l}\text { Third postoperative } \\
\text { questionnaire (T3) }\end{array}$ & $\begin{array}{l}12 \text { months after } \\
\text { surgery }\end{array}$ & $\begin{array}{l}\text { To integrate information } \\
\text { on outcomes and map } \\
\text { the postoperative care } \\
\text { pathway; to identify } \\
\text { experiential factors } \\
\text { affecting the outcomes }\end{array}$ & $\begin{array}{l}\text { Experience with } \\
\text { services }\end{array}$ & $\begin{array}{l}\text { Rehabilitation pathway. } \\
\text { Home care. } \\
\text { Recourse to private and out-of-pocket care services. } \\
\text { Follow-up visits. } \\
\text { Role of the general practitioner. } \\
\text { Open-ended question on the care pathway after } \\
\text { surgery. }\end{array}$ \\
\hline
\end{tabular}

GP, general practitioner; PREM, patient-reported experience measure. 
Table 3 Additional data collected automatically or by professionals with the Orthopaedic PROMs and PREMs Observatory initiative by phase

\begin{tabular}{|c|c|c|c|}
\hline Phase & Timing of administration & Dimensions & Items \\
\hline \multirow[t]{2}{*}{ Enrolment } & \multirow[t]{2}{*}{$\begin{array}{l}\text { Before surgery (at surgical } \\
\text { indication or preadmission) }\end{array}$} & $\begin{array}{l}\text { Identifying information of } \\
\text { patients }\end{array}$ & $\begin{array}{l}\text { Name and surname. } \\
\text { Contacts. }\end{array}$ \\
\hline & & $\begin{array}{l}\text { Clinically related } \\
\text { information }\end{array}$ & $\begin{array}{l}\text { Comorbidities. } \\
\text { Use of psychotropic drugs. } \\
\text { Use of aids. }\end{array}$ \\
\hline After surgery & Within 30 days after surgery & $\begin{array}{l}\text { Information on the } \\
\text { procedure }\end{array}$ & $\begin{array}{l}\text { Date of surgery. } \\
\text { Characteristics of the implant. } \\
\text { Type of surgical access. } \\
\text { Type of surgical procedure (ie, robotic surgery). }\end{array}$ \\
\hline
\end{tabular}

All questionnaires include a question about how patients perceive their health status, with respect to the past week. The question is 'In general, how would you rate your health status in the last week? Excellent/very good/ good/fair/bad'. In each questionnaire, patients are also asked if there is someone supporting them in accessing and answering the questionnaire because it could influence the respondent similarly to an interviewer.

The digital system automatically collects the date of enrolment and questionnaires' completion, which can be used to adjust results. The healthcare professionals or their staff can report additional information on the web platform (table 3).

\section{PHASES OF DEVELOPMENT AND IMPLEMENTATION}

\section{Stakeholders' involvement}

The OPPO was designed together with healthcare professionals and patients.

Two preliminary meetings have been organised in March and May 2017, with around 10 representatives of the orthopaedic professionals, identified and invited by the managers of the Tuscan healthcare organisations. The two meetings were aimed at outlining the project, sharing the patients' eligibility criteria, and defining methods of administration as well as timing and contents of the questionnaires.

From May to October 2017, several site visits were organised with orthopaedic operative units' managers and professionals at all public hospitals where THR and TKR interventions are carried out in Tuscany. During the site visits, the aforementioned topics were again discussed and shared. The visits were also aimed at mapping the different phases of the patient's pathway, from the preadmission to the postoperative pathway. This mapping was useful to better design the enrolment phase and will turn useful to evaluate variations in providing services. The professionals' feedback was fundamental to move from a unique to a customisable enrolment procedure. Since the digital process of recruitment through the web platform collects the dates of enrolment, it is possible to detect variations and adjust PROMs and PREMs data. The site visits are ongoing, with the aim to recruit additional operative units and to continuously motivate professionals.

Health professionals are involved in the analysis and the discussion of data, both individually and collectively during specific workshops organised at least twice per year.

\section{Patient and public involvement}

Patients were also involved in designing the OPPO. In September 2017, 20 patients waiting for THR or TKR surgery at the Viareggio Hospital participated in a meeting. The OPPO initiative was presented and their feedback on acceptability and burden was collected. A T0 test was also performed to check questions' readability and comprehensibility, and the accessibility of the questionnaire, also depending on the device (ie, mobile phone and tablet). Improvements were subsequently made based on the suggestions received.

\section{Development}

The OPPO is digitally managed, following three phases (figure 3).

At baseline, orthopaedic professionals and their collaborators identify eligible patients that meet the inclusion criteria. Eligible patients are informed and, if they agree to participate, the staff collects their information on a web platform that automatically invites enrolled patients.

The link to the preoperative questionnaire is sent by SMS and/or email within 24 hours after enrolment. As anticipated, since there is an extensive variation of waiting 


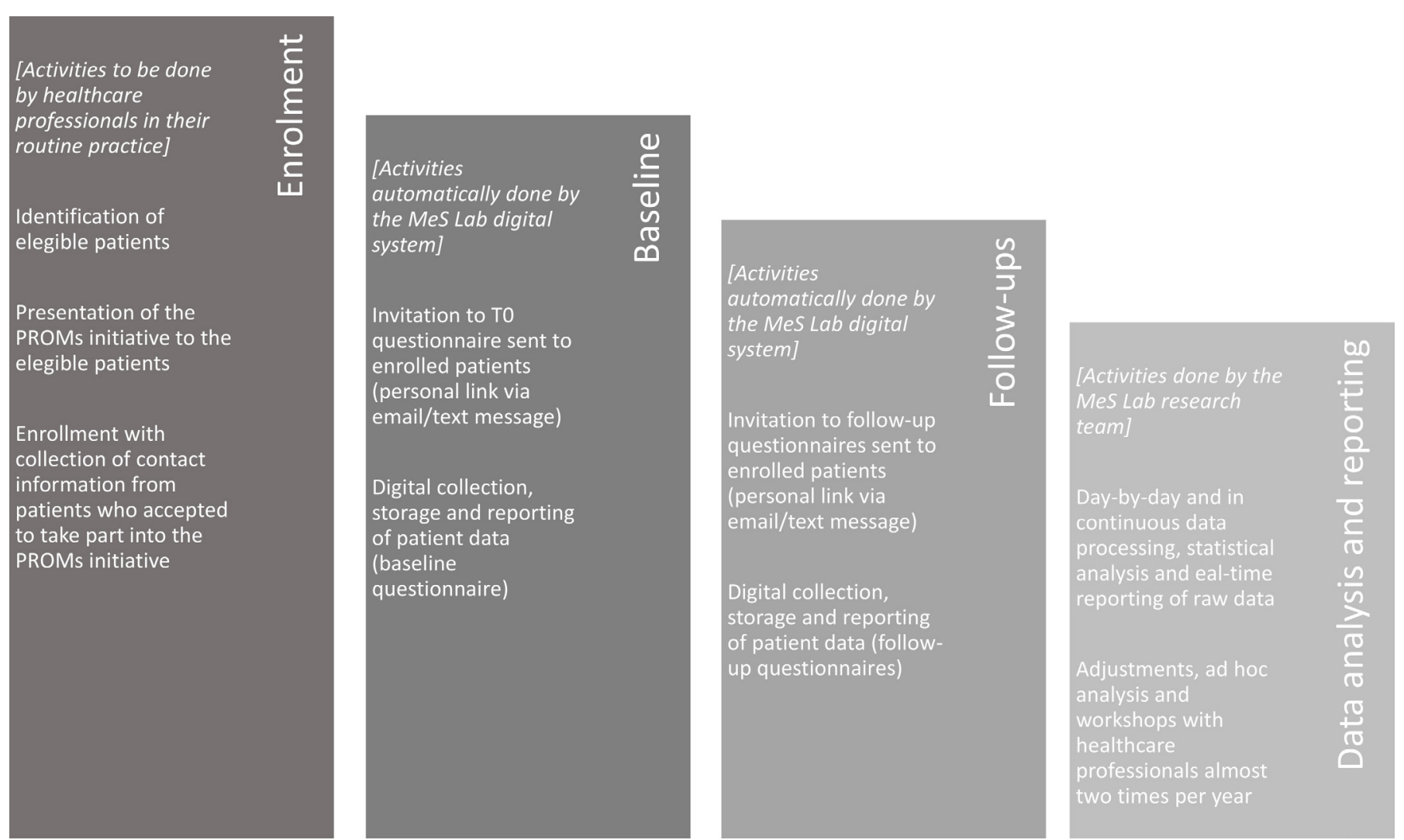

Figure 3 Orthopaedic PROMs and PREMs Observatory flowchart showing the phases (baseline, follow-up and data analysis) currently performed in continuously and routinely. PROM, patient-reported outcome measure.

times for THR and TKR between and within healthcare organisations, the preoperative questionnaire can be administered at different timepoints before surgery.

The first follow-up postoperative questionnaire (T1) is administered 30 days after surgery. Health professionals indicate the surgery date within 30 days from surgery, using the same web platform of the enrolment. An email reminder is automatically sent to enrollers 15 days from the last enrolment without a surgery date. The second (T2) and third (T3) follow-up postoperative questionnaires are automatically sent out by the web platform, according to the surgery date reported by professionals.

Healthcare professionals can access the web platform to monitor the surveys by checking their enrolment and response rates.

\section{ENDPOINTS AND ANALYSES}

The endpoints of interest are measured through the OHS and OKS scores, the difference between scores over time (delta score), and the improvement index. The measure of the overall health status will be reported by a score on a 0-100 scale. Other outcomes include complications, adverse events and hospital readmissions. The PREM scales will be used to measure the care outcomes in terms of patient experience and satisfaction with healthcare services, along the care pathway (table 2). The different variables collected in the study, such as sociodemographic conditions, lifestyle, type of prosthesis and surgical unit, will be used to improve the comparability of PROM and PREM data, refining the risk adjustment algorithm of the results.

Statistical analyses will be separately performed for patients undergoing THR and TKR.

Patient-reported outcomes will be analysed through various methodologies, including regression models, generalised linear models and structural equation models. In particular, the analysis will be oriented to detect outcomes' trends and evaluate variations. Furthermore, the determinants of the outcomes will be explored by investigating associations between PROM scores and PREM, identifying different models of service delivery along the pathway and their association with the PROM score, and integrating PROMs with outcome measures from administrative and/or clinical source. The choice of administering the first follow-up questionnaire 30 days after surgery relies on the willingness to acquire relevant and prompt PREM data about hospital stay. Although this time period could be an early stage of evaluation for the intermediate outcome, with these timepoints of PROM administration, the patients' recovery monitoring is more detailed and granular. This allows detection of eventual effects on the quality of life and functionalities produced by a different organisation or provision of healthcare services during hospitalisation and just after discharge. PROM and PREM data will be integrated with data from the PES of Tuscany. ${ }^{21} 29$ 
By using dates of enrolment and questionnaire completion, the effect of time passed since the T0 completion and surgery will be evaluated, especially in terms of impact on the difference in outcomes between preoperative and postoperative questionnaires.

Researchers will compute and test the effect of sample attrition rates for each T(i) of both THR and TKR by investigating if attrition rate over time is missing randomly or if it is associated with a systematic error, thus producing biased estimations. Sensitivity analyses will be conducted to determine the potential presence of bias in follow-up responses related to patients' conditions. Indeed, previous research showed that the patients' interest, time availability and health status can affect their willingness and ability to participate in longitudinal studies, ${ }^{45960}$ and therefore the sensitivity analyses aim at ensuring that responses are not biased by patients' characteristics and conditions (eg, sex, age, education level, comorbidities, health condition at baseline, typology of intervention and presence of a caregiver).

The representativeness of respondents to the OPPO surveys will be investigated. The respondents' characteristics will be compared with the characteristics of all the patients who underwent the same surgery in the same period in the same hospitals. The characteristics of the population will be extracted from administrative data related to the discharge events (the dataflow is called 'scheda di dimissione ospedaliera' or hospital discharge form). Patients' demographic information (ie, sex, age, education level and citizenship) will be used for this comparison, performed through t-tests, and eventually using other sensitivity analyses and matching techniques. Clinical variables (such as comorbidities) and surgical details (ie, type of surgical procedure and technique, and type of prosthesis) will be considered for additional comparative analyses. If any significant difference will emerge, data will be weighted at the hospital level, after a multistratification of both respondents and population of reference, considering the significant variables of analysis. If a negligible deviation will emerge, a further sensitivity analysis will be performed before risk adjusting by performing multilevel models of regression at hospital level and using significant variables.

\section{ETHICS AND DISSEMINATION}

Ethics and privacy issues were addressed for the PROMS and PREMs observatory, with the Tuscan regional office that provides support for health research and clinical trials, supervises the protection of personal data in the health sector and supports the activities of the ethics committees. By collaborating with this office, researchers prepared the following formal documents: participant information sheet, copy of the questionnaires, list of data collected by professionals during enrolment, study protocol and data management plan. These documents were submitted to the evaluation of the four Tuscan ethics committees, obtaining the nihil obstat from all regional ethics committees of Tuscany region, between November and December 2017 (the paediatric ethics committees provided a certification of notice). The data protection officers of all healthcare organisations, together with the appointed aforementioned office of Tuscany region, framed the initiative in a legal framework that refers to the national guidelines of the Italian Data Protection Authority, ${ }^{61}$ the Legislative Decree 196/2003 (Art. 2 sexies, comma 2, letter V) and the regional law that implements the aforementioned legislative decree and regulates patient surveys (Decree of the President of Tuscany Region number 6/R/2013, sheet 12 appendix A, and sheet 39 appendix B). Accordingly, PROMs were considered regulated as the other patient surveys, such as PREMs. ${ }^{62}$ Indeed, starting from 2019, the informed consent was not required anymore for PROMs. Patients are accurately informed, also using a specific informative document, that they are free to participate or not in the survey and can drop out at any time.

Patient data are stored in separate datasets. Names and surnames of enrolled patients are collected by healthcare staff and saved in a dataset, which is visible on the web platform to the enrollers. The web platform access is regulated by credentials (staff must login with username and password) and after a previous authorisation. Survey data are saved in separate datasets and are linked to the enrolment dataset by a token. The token is a unique code automatically generated by the web platform. The token allows sending of questionnaires to patients without identifying them. The survey datasets do not contain any patient identification data. The name and surname, as well as contact data, are deleted at the completion of the last questionnaire, or when the last reminder is sent by email or SMS, or when the patient drops out from the survey. All datasets are stored on the server of Sant'Anna School, in Pisa, Italy. The data collection and storage systems (ie, web platform and server) are set according to the European Data Protection Directive. ${ }^{63}$

The findings will be reported for publication in peer-reviewed journals and presented to appropriate conferences. The results will be returned exclusively in anonymised and aggregated form.

Real-time updated PROM and PREM raw results are available on the online platform, anonymised and aggregated, in graphs and tables, if a threshold of at least 15 respondents has been reached. They can be visualised by the professionals and operative units' managers involved in the initiative, as well as by their hospitals and healthcare organisations' managers. Data analysis is periodically performed by the research team of the MeS Laboratory, and PROM and PREM results are discussed with orthopaedic professionals at least every 6 months, through workshops. During the workshops, results of all teams are benchmarked, and they are used for discussion among clinicians. The aggregated performance data of each unit are transparently disclosed to all clinicians, while more detailed anonymised and aggregated data are available to each clinician about her/his patients on the web 
platform. The availability of data make it possible for the clinicians to match and compare results and scales reported by patients with those collected by clinicians, indirectly by using data from different sources, or directly by collaborating with the research team of the MeS Laboratory. The data are periodically disseminated via reports and presented to Tuscan healthcare managers and policymakers. Data will be used to yearly compute indicators included in the PES of Tuscany, which is publicly available online on the MeS Laboratory website (https://performance.santannapisa.it/pes).

The protocol will be disseminated via the study web page available online (https://www.santannapisa.it/it/ricerca/ progetti/indagini-proms-nella-chirurgia-ortopedica).

\section{DISCUSSION}

This protocol describes the first regional-level digital and continuous PROMs and PREMs observatory on THR and TKR in Italy. Patient-reported indicators after elective joint replacement are generally not available at the provider level in Italy, despite this highly common surgical procedure presenting variable outcomes across patient groups, providers and geographical areas. ${ }^{606465}$ The OPPO will provide (1) a more comprehensive understanding of outcomes and effectiveness, integrating the clinical perspective with the patients' perspective; (2) prompt data for improving services; and (3) information to identify best practices about the organisation and management of the whole care pathway. By integrating PROMs-based indicators from the OPPO into the healthcare PES, policymakers and managers will be able to better allocate or reallocate resources, ${ }^{18}$ according to a value model and a population-based approach to care. ${ }^{296667}$ This kind of PROM use is not well established and several challenges emerged in other experiences, like in the English PROMs initiative. ${ }^{68}$ Timeliness of data reporting was one of the barriers in patient data use,${ }^{68}$ while one of the strengths of the OPPO is the real-time reporting of data to providers. This initiative will provide evidence on the impact that a prompt data sharing can have on the actual use of PROMs data by providers at different levels.

Data from the OPPO will contribute to longitudinally investigate trends of PROMs and PREMs by providing an integrated pathway vision, from the patient's point of view. Since measurements and assessments are usually performed following the perspective of healthcare organisations or clinicians, this initiative will overcome the consequent 'silo vision', following instead a multiprovider and cross-setting patient pathway approach. ${ }^{29} 66$ Findings from the OPPO will provide additional evidence on the effectiveness of different postoperative pathways. For instance, there is a mixed evidence on whether, when and how the rehabilitation affects the THR and TKR outcomes. $^{1069-74}$

Another strength of the OPPO initiative is the combined collection of PROMs and PREMs. Previous studies showed that there is a positive and overtime consistent association between patient experience and effectiveness of THR and TKR procedures. ${ }^{75}$ Data from the OPPO will contribute to increase the consistency of scientific evidence on this topic.

The continuity of the PROMs and PREMs collection is among the strengths of this initiative because it allows testing and comparing different measurement scales over time.

Another important strength is represented by the affordability of the initiative in terms of costs. The digital data collection and reporting are low cost and easy to use, and can offer multiple additional functions, especially if compared with postal or phone surveys. ${ }^{22}$ Despite older people being more and more familiar with the digital technologies, ${ }^{42} 43$ elderly patients can have difficulties in participating in a digital survey. Therefore, if patients need and want it, caregivers are also informed and involved in the enrolment phase. This can mitigate the risk of a low participation of elderly patients.

This initiative has some limitations. First, the OPPO is multicentric, but it has been implemented in only one Italian region. Nevertheless, the methodology adopted in the OPPO guarantees the technical feasibility of an extension with affordable costs. Second, at this stage, the study is built in a way that is not suitable for people with severe cognitive impairments. Future research should give more space to the development of specific methodologies aimed at involving these fragile patients. Another limitation is that the system is provider-dependent: clinicians and their staff are in charge of informing and enrolling patients. Thus, a key critical aspect is represented by an extensive and periodic involvement and training of professionals. On the one hand, their engagement can motivate them, enhance their interest and support the use of PROM and PREM data in 'real-life' contexts. ${ }^{37}$ On the other hand, some additional managerial levers are needed to enhance professionals' participation: with regard to the OPPO, yearly indicators on enrolment rates and preoperative response rates, with specific targets, were introduced in the Tuscan PES.

\section{Twitter Sabina De Rosis @SabinaDeRosis}

Acknowledgements The authors thank all the orthopaedic professionals and their staff involved in the PROMs and PREMs Observatory. The authors are also grateful to the regional administrators of Tuscany and their staff, as well as the managers and the healthcare personnel of participating healthcare organisations, who made possible the implementation of the initiative. The authors also gratefully acknowledge patients participating in the Observatory and their caregivers. The authors thank all the MeS Laboratory researchers for their valuable support. The authors are also grateful to Tommaso Grillo Ruggieri for his contribution in the first phases of the design and implementation of the initiative.

Contributors SN conceived the idea of the PROMs Observatory and is the research responsible and supervisor. SDR is the scientific director and coordinator of the initiative. SN, SDR, DAL and FP were responsible for the study design and implementation. MM collaborated in the study design, including the early involvement of patients, and gave support in the results' interpretation. SDR, DAL and FP will be responsible for the analysis of results. SDR drafted the manuscript. DAL and FP contributed to writing the manuscript. SN and MM critically reviewed the manuscript. All contributors read and approved this version submitted for publication to BMJ Open. 
Funding The Orthopaedic PROMs and PREMs Observatory (OPPO) was commissioned and funded by the 'Direzione Diritti di cittadinanza e coesione sociale' of Tuscany Region (Regional Act n.4182 adopted on 3 April 2017). The funding body collaborated in the design of the surveys by discussing the questionnaires and the administration methodology with the research team, and in the interpretation of the preliminary results of the OPPO

Competing interests MM is an orthopaedic surgeon from one of the local health authorities participating to the initiative.

Patient and public involvement Patients and/or the public were involved in the design, conduct, reporting or dissemination plans of this research. Refer to the Methods section for further details.

Patient consent for publication Not applicable.

Provenance and peer review Not commissioned; externally peer reviewed.

Data availability statement No data are available.

Open access This is an open access article distributed in accordance with the Creative Commons Attribution Non Commercial (CC BY-NC 4.0) license, which permits others to distribute, remix, adapt, build upon this work non-commercially, and license their derivative works on different terms, provided the original work is properly cited, appropriate credit is given, any changes made indicated, and the use is non-commercial. See: http://creativecommons.org/licenses/by-nc/4.0/.

\section{ORCID iDs}

Sabina De Rosis http://orcid.org/0000-0002-8781-401X

Francesca Pennucci http://orcid.org/0000-0002-0580-1435

Daniel Adrian Lungu http://orcid.org/0000-0002-8612-8384

Sabina Nuti http://orcid.org/0000-0002-2093-1828

\section{REFERENCES}

1 Calvert M, Kyte D, Mercieca-Bebber R, et al. Guidelines for inclusion of patient-reported outcomes in clinical trial protocols: the SPIRITPRO extension. JAMA 2018;319:483-94.

2 Palazzo C, Nguyen C, Lefevre-Colau M-M, et al. Risk factors and burden of osteoarthritis. Ann Phys Rehabil Med 2016;59:134-8.

3 Chen C, Haddad D, Selsky J, et al. Making sense of mobile health data: an open architecture to improve individual- and populationlevel health. J Med Internet Res 2012;14:e112.

4 Cecchi F, Mannoni A, Molino-Lova R, et al. Epidemiology of hip and knee pain in a community based sample of Italian persons aged 65 and older. Osteoarthritis Cartilage 2008;16:1039-46.

5 Salute Mdella. Ufficio 6 DG della Programmazione sanitaria (2019) Rapporto annuale sull'attivit di ricovero ospedaliero. Dati SDO, 2017.

6 Kamaruzaman $\mathrm{H}$, Kinghorn P, Oppong R. Cost-Effectiveness of surgical interventions for the management of osteoarthritis: a systematic review of the literature. BMC Musculoskelet Disord 2017:18:183.

7 Tian W, DeJong G, Brown M, et al. Looking upstream: factors shaping the demand for postacute joint replacement rehabilitation. Arch Phys Med Rehabil 2009;90:1260-8.

8 Allen KD, Golightly YM. State of the evidence. Curr Opin Rheumatol 2015;27:276-83.

9 Chen A, Gupte C, Akhtar K, et al. The global economic cost of osteoarthritis: how the UK compares. Arthritis 2012;2012:698709.

10 Pathak S, Ganduglia CM, Awad SS, et al. Is post-discharge rehabilitation timing associated with 90 -day readmission in primary total joint arthroplasty? Hss J 2019;15:234-40.

11 Barbieri A, Vanhaecht K, Van Herck P, et al. Effects of clinical pathways in the joint replacement: a meta-analysis. BMC Med 2009;7:32.

12 Westby MD, Klemm A, Li LC, et al. Emerging role of quality indicators in physical therapist practice and health service delivery. Phys Ther 2016;96:90-100.

13 Westby MD, Brittain A, Backman CL. Expert consensus on best practices for post-acute rehabilitation after total hip and knee arthroplasty: a Canada and United States Delphi study. Arthritis Care Res 2014;66:411-23.

14 Dreinhöfer KE, Dieppe P, Stürmer T, et al. Indications for total hip replacement: comparison of assessments of orthopaedic surgeons and referring physicians. Ann Rheum Dis 2006;65:1346-50.

15 Ten Have IA, van den Bekerom MP, van Deurzen DF, et al. Role of decision AIDS in orthopaedic surgery. World J Orthop 2015;6:864-6.

16 Lange T, Rataj E, Kopkow C, et al. Outcome assessment in total knee arthroplasty: a systematic review and critical appraisal. J Arthroplasty 2017;32:653-65.
17 Black N. Patient reported outcome measures could help transform healthcare. BMJ 2013;346:f167.

18 De Rosis S, Pennucci F, Nuti S. From the experience and outcome measurement to the health professionals engagement. Micro \& Macro Marketing 2019;1:493-520.

19 Pushing the boundaries: frontiers of quality of life research. Abstracts of the 18th annual conference of the International Society for quality of life research. October 26-29, 2011. Denver, Colorado, USA. Qual Life Res 2012;20 Suppl 1:2-117.

20 Snyder CF, Aaronson NK. Use of patient-reported outcomes in clinical practice. Lancet 2009;374:369-70.

21 Nuti S, Noto G, Vola F. Let's play the patients music: A new generation of performance measurement systems in healthcare. Management Decision 2018;56:2252-72.

22 Coulter A, Fitzpatrick R, Cornwell J. Measures of patients' experience in hospital: purpose, methods and uses. King's Fund London, 2009.

23 Gibbons E, Black N, Fallowfield L, et al. Patient-reported outcome measures and the evaluation of services. NIHR Journals Library, 2016.

24 Canadian Institute for Health Information. Health outcomes of care: an idea whose time has come. Ottawa, Ontario, 2012.

25 Prodinger B, Taylor P. Improving quality of care through patientreported outcome measures (PROMs): expert interviews using the NHS PROMs programme and the Swedish quality registers for knee and hip arthroplasty as examples. BMC Health Serv Res 2018;18:87.

26 Baker PN, Deehan DJ, Lees D, et al. The effect of surgical factors on early patient-reported outcome measures (PROMs) following total knee replacement. J Bone Joint Surg Br 2012;94:1058-66.

27 Peters M, Crocker H, Jenkinson C, et al. The routine collection of patient-reported outcome measures (PROMs) for longterm conditions in primary care: a cohort survey. BMJ Open 2014:4:e003968.

28 OECD. Recommendations to OECD Ministers of Health from the High Level Reflection Group on the Future of Health Statistics - Strengthening the international comparison of health system performance through patient-reported indicators 2017

29 Nuti S, De Rosis S, Bonciani M, et al. Rethinking healthcare performance evaluation systems towards the People-Centredness approach: their pathways, their experience, their evaluation. Healthc Pap 2017:17:56-64.

30 Flott KM, Graham C, Darzi A, et al. Can we use patient-reported feedback to drive change? The challenges of using patient-reported feedback and how they might be addressed. BMJ Qual Saf 2017;26:502-7.

31 Gleeson H, Calderon A, Swami V, et al. Systematic review of approaches to using patient experience data for quality improvement in healthcare settings. BMJ Open 2016;6:e011907.

32 Slawomirski L, van den Berg M, Karmakar-Hore S. Patient-Reported indicator survey (Paris): aligning practice and policy for better health outcomes. World Medical Journal 2018:64:8-13.

33 OECD/EU. Health at a glance 2019: OECD indicators, 2019.

34 Chen J, Ou L, Hollis SJ. A systematic review of the impact of routine collection of patient reported outcome measures on patients, providers and health organisations in an oncologic setting. BMC Health Serv Res 2013;13:211.

35 Ghilli M, Mariniello MD, Camilleri V, et al. Proms in post-mastectomy care: patient self-reports (BREAST-Q ${ }^{\mathrm{TM}}$ ) as a powerful instrument to personalize medical services. Eur J Surg Oncol 2020;46:1034-40.

36 Ferrè F, De Rosis S, Murante AM, et al. Systematic and continuous collection of patient-reported outcomes and experience in women with cancer undergoing mastectomy and immediate breast reconstruction: a study protocol for the Tuscany region (Italy). BMJ Open 2021;11:e042235.

37 Lungu DA, Pennucci F, De Rosis S, et al. Implementing successful systematic patient reported outcome and experience measures (PROMs and PREMs) in robotic oncological surgery-The role of physicians. Int J Health Plann Manage 2020;35:773-87.

38 Gilmore KJ, Pennucci F, De Rosis S, et al. Value in healthcare and the role of the patient voice. Healthc Pap 2019;18:28-35.

39 Pennucci F, De Rosis S, Nuti S. Can the jointly collection of PROMs and PREMs improve integrated care? the changing process of the assessment system for the hearth failure path in Tuscany region. Int $J$ Integr Care 2019;19:421.

40 Pennucci F, De Rosis S, Passino C. Piloting a web-based systematic collection and reporting of patient-reported outcome measures and patient-reported experience measures in chronic heart failure. BMJ Open 2020;10:e037754.

41 Bonciani M, De Rosis S, Vainieri M. Mobile health intervention in the maternal care pathway: protocol for the impact evaluation of hAPPyMamma. JMIR Res Protoc 2021;10:e19073. 
42 Remillard ML, Mazor KM, Cutrona SL, et al. Systematic review of the use of online questionnaires of older adults. J Am Geriatr Soc 2014;62:696-705.

43 Hoque R, Sorwar G. Understanding factors influencing the adoption of mHealth by the elderly: an extension of the UTAUT model. Int $J$ Med Inform 2017;101:75-84.

44 van Gelder MMHJ, Bretveld RW, Roeleveld N. Web-Based questionnaires: the future in epidemiology? Am J Epidemiol 2010;172:1292-8.

45 Ackerman IN, Kemp JL, Crossley KM, et al. Hip and knee osteoarthritis affects younger people, too. J Orthop Sports Phys Ther 2017;47:67-79.

46 Smith TO, Gilbert AW, Sreekanta A, et al. Enhanced rehabilitation and care models for adults with dementia following hip fracture surgery. Cochrane Database Syst Rev 2020;2:CD010569.

47 Morton SMB, Bandara DK, Robinson EM, et al. In the 21st century, what is an acceptable response rate? Aust $N Z \mathrm{~J}$ Public Health 2012;36:106-8.

48 Galea S, Tracy M. Participation rates in epidemiologic studies. Ann Epidemiol 2007;17:643-53.

49 Gustavson K, von Soest T, Karevold E, et al. Attrition and generalizability in longitudinal studies: findings from a 15-year population-based study and a Monte Carlo simulation study. BMC Public Health 2012;12:918.

50 Dawson J, Fitzpatrick R, Frost S, et al. Evidence for the validity of a patient-based instrument for assessment of outcome after revision hip replacement. J Bone Joint Surg Br 2001;83:1125-9.

51 Murray DW, Fitzpatrick R, Rogers K, et al. The use of the Oxford hip and knee scores. J Bone Joint Surg Br 2007;89:1010-4.

52 Judge A, Arden NK, Price A, et al. Assessing patients for joint replacement: can pre-operative Oxford hip and knee scores be used to predict patient satisfaction following joint replacement surgery and to guide patient selection? J Bone Joint Surg $\mathrm{Br}$ 2011;93:1660-4.

53 Dawson J, Fitzpatrick R, Carr A, et al. Questionnaire on the perceptions of patients about total hip replacement. J Bone Joint Surg Br 1996;78-B:185-90.

54 Dawson J, Fitzpatrick R, Murray D, et al. Questionnaire on the perceptions of patients about total knee replacement. J Bone Joint Surg Br 1998;80:63-9.

55 Padua R, Zanoli G, Ceccarelli E, et al. The Italian version of the Oxford 12-Item knee Questionnaire-cross-cultural adaptation and validation. Int Orthop 2003;27:214-6.

56 Martinelli N, Longo UG, Marinozzi A, et al. Cross-Cultural adaptation and validation with reliability, validity, and responsiveness of the Italian version of the Oxford hip score in patients with hip osteoarthritis. Qual Life Res 2011;20:923-9.

57 Dawson J, Fitzpatrick R, Murray D, et al. Comparison of measures to assess outcomes in total hip replacement surgery. Qual Health Care 1996;5:81-8.

58 Ostendorf M, van Stel HF, Buskens E, et al. Patient-Reported outcome in total hip replacement. A comparison of five instruments of health status. J Bone Joint Surg Br 2004;86:801-8.
59 Gaertner B, Seitz I, Fuchs J, et al. Baseline participation in a health examination survey of the population 65 years and older: who is missed and why? BMC Geriatr 2016;16:21.

60 Grassi A, Golinelli D, Tedesco D, et al. Patient-Reported outcome measures (PROMs) after elective hip, knee and shoulder arthroplasty: protocol for a prospective cohort study. BMC Musculoskelet Disord 2019;20:374.

61 Italian Data Protection Authority. Guidelines on processing personal data to perform customer satisfaction surveys in the health care sector, 2011.

62 De Rosis S, Cerasuolo D, Nuti S. Using patient-reported measures to drive change in healthcare: the experience of the digital, continuous and systematic PREMs observatory in Italy. BMC Health Serv Res 2020;20:315.

63 European Parliament and Council. Regulation (EU) of the European Parliament and of the Council on the protection of natural persons with regard to the processing of personal data and on the free movement of such data, and repealing directive 95/46/EC (General data protection regulation) 2016.

64 Lingard EA, Katz JN, Wright EA, et al. Predicting the outcome of total knee arthroplasty. The Journal of Bone \& Joint Surgery 2004;86:2179-86.

65 Dieppe $\mathrm{P}$, Judge A, Williams S, et al. Variations in the preoperative status of patients coming to primary hip replacement for osteoarthritis in European orthopaedic centres. BMC Musculoskelet Disord 2009;10:19.

66 Nuti S, Noto G, Vola F, et al. Let's play the patients music: A new generation of performance measurement systems in healthcare. Management Decision, 2018.

67 Gray M, Jani A. Promoting triple value healthcare in countries with universal healthcare. Healthc Pap 2016;15:42-8.

68 Black N, Burke L, Forrest CB, et al. Patient-Reported outcomes: pathways to better health, better services, and better societies. Qual Life Res 2016;25:1103-12.

69 Austin MS, Urbani BT, Fleischman AN, et al. Formal physical therapy after total hip arthroplasty is not required: a randomized controlled trial. J Bone Joint Surg Am 2017;99:648-55.

70 Husted H. Fast-Track hip and knee arthroplasty: clinical and organizational aspects. Acta Orthop Suppl 2012;83:1-39.

71 Roos EM. Effectiveness and practice variation of rehabilitation after joint replacement. Curr Opin Rheumatol 2003;15:160-2.

72 Lowe CJM, Davies L, Sackley CM, et al. Effectiveness of landbased physiotherapy exercise following hospital discharge following hip arthroplasty for osteoarthritis: an updated systematic review. Physiotherapy 2015;101:252-65.

73 Galbraith AS, McGloughlin E, Cashman J. Enhanced recovery protocols in total joint arthroplasty: a review of the literature and their implementation. Ir J Med Sci 2018;187:97-109.

74 Artz N, Elvers KT, Lowe CM, et al. Effectiveness of physiotherapy exercise following total knee replacement: systematic review and meta-analysis. BMC Musculoskelet Disord 2015;16:15.

75 Black N, Varaganum M, Hutchings A. Relationship between patient reported experience (PREMs) and patient reported outcomes (PROMs) in elective surgery. BMJ Qual Saf 2014;23:534 